\title{
Successful implementation of an Enhanced Recovery After Surgery (ERAS) protocol reduces nausea and vomiting in patients after infratentorial craniotomy for tumour resection: a randomized controlled trial.
}

\section{Dan Lu(Former Corresponding Author)}

Xi'an international medical center https://orcid.org/0000-0001-8297-4383

\section{Yuan Wang}

Xi'an Tangdu Hospital of No4 Military Medical University

Tianzhi Zhao

Xi'an Tangdu Hospital of No4 Military Medical University

Bolin Liu

xi'an international medical center

Lin Ye

Xi'an Tangdu Hospital of No4 Military Medical University

Lanfu Zhao

Xi'an Tangdu Hospital of No4 Military Medical University

\section{Binfang Zhao}

Xi'an Tangdu Hospital of No4 Military Medical University Mingjuan Li

Xi'an Tangdu Hospital of No4 Military Medical University

\section{Lin Ma}

Xi'an Tangdu Hospital of No4 Military Medical University

Zhengmin Li

Xi'an Tangdu Hospital of No4 Military Medical University Jiangtao Niu

Xi'an Tangdu Hospital of No4 Military Medical University

\section{Wenhai LV}

Xi'an Tangdu Hospital of No4 Military Medical University

\section{Yufu Zhang}

Xi'an Tangdu Hospital of No4 Military Medical University

\section{Tao Zheng}

xi'an international medical center 


\section{Yafei Xue}

Xi'an Tangdu Hospital of No4 Military Medical University

\section{Lei Chen}

xi'an international medical center

\section{Long Chen}

Xi'an Tangdu Hospital of No4 Military Medical University

\section{Xude Sun}

Xi'an Tangdu Hospital of No4 Military Medical University

\section{Guodong Gao}

Xi'an Tangdu Hospital of No4 Military Medical University

\section{Bo Chen}

Shaanxi Provincial People's Hospital

Shiming He(New Corresponding Author) ( $\nabla$ he-shiming@163.com )

Xi'an International Medical Center

\section{Research article}

Keywords: enhanced recovery after surgery (ERAS), postoperative nausea and vomiting (PONV), infratentorial craniotomy, anxiety, sleep quality

Posted Date: March 16th, 2020

DOI: https://doi.org/10.21203/rs.2.15916/v3

License: (c) (1) This work is licensed under a Creative Commons Attribution 4.0 International License. Read Full License

Version of Record: A version of this preprint was published at BMC Neurology on April 22nd, 2020. See the published version at https://doi.org/10.1186/s12883-020-01699-z.

\section{EDITORIAL NOTE:}

24 January 2022: The published version of this preprint was retracted from BMC Neurology on 17 December 2020. The retraction notice can be found here. Research Square has withdrawn this preprint. 


\section{Abstract}

Objectives : Infratentorial craniotomy patients have a high incidence of postoperative nausea and vomiting (PONV). Enhanced Recovery After Surgery (ERAS) protocols have been shown in multiple surgical disciplines to improve outcomes, including reduced PONV. However, very few studies have described the application of ERAS to infratentorial craniotomy. The aim of this study was to examine whether our ERAS protocol for infratentorial craniotomy could improve PONV.

Methods : We implemented an evidence-based, multimodal ERAS protocol for patients undergoing infratentorial craniotomy. A total of 105 patients who underwent infratentorial craniotomy were randomized into either the ERAS group $(n=50)$ or the control group $(n=55)$. Primary outcomes were the incidence of vomiting, nausea score, and use of rescue antiemetic during the first $72 \mathrm{~h}$ after surgery. Secondary outcomes included postoperative anxiety level, sleep quality, and complications.

Results: Over the entire $72 \mathrm{~h}$ post-craniotomy observation period, the cumulative incidence of vomiting was significantly lower in the ERAS group than in the control group. Meanwhile, the incidence of vomiting was significantly lower in the ERAS group on postoperative days (PODs) 2 and 3. Notably, the proportion of patients with mild nausea (VAS 0-4) was higher in the ERAS group as compared to the control group on PODs 2 or 3 . Additionally, the postoperative anxiety level and quality of sleep were significantly better in the ERAS group.

Conclusion: Successful implementation of our ERAS protocol in infratentorial craniotomy patients could attenuate postoperative anxiety, improve sleep quality, and reduce the incidence of PONV, without increasing the rate of postoperative complications.

\section{Background}

Neurosurgical patients are generally considered to have high risk of postoperative nausea and vomiting (PONV) according to the consensus guidelines for managing PONV[1]. Moreover, the incidence of PONV is more frequent after infratentorial procedures than supratentorial procedures[2]. Postoperative vomiting is not only unpleasant, but may also cause serious complications in neurosurgical patients, such as dehydration, electrolyte disturbances, aspiration and alkalosis. Poor control of PONV is associated with worse quality of life outcomes in the postoperative period, and is one of the most common reasons for prolonged hospitalization, which can contribute to healthcare costs.

Previous reports indicated that significant improvements in postoperative recovery can be achieved by implementing a standardized protocol of evidence-based treatments over the entire perioperative period[3, 4]. This concept of enhanced recovery after surgery (ERAS) was originally conceptualized by Kehlet to improve functional outcomes after surgery and decrease perioperative morbidity [5, 6]. ERAS protocols have been widely utilized in the perioperative period in several surgical fields such as colorectal surgery, urological surgery, and orthopedic surgery[7-9]. However, to the best of our knowledge, application of an ERAS protocol for infratentorial craniotomy has not yet been reported. Hagan et al. proposed some key 
components of ERAS applicable to craniotomy based on evidence available from other surgical specialties[10].

Based on this preliminary protocol and our own institutional experience, we presented a novel ERAS protocol for patients undergoing craniotomy at Tangdu hospital (Supplementary file). The aim of the present study was to prospectively evaluate whether this ERAS protocol significantly improved PONV after infratentorial craniotomy.

\section{Methods}

Study criteria

The present study was performed at Tangdu Hospital, which is affiliated with the Fourth Military Medical University. This study was approved by the institutional human research and ethics committee of Tangdu Hospital. This randomized control trial (RCT) was registered at the Chinese Trial Registry (ChiCTR-INR16009662). In June 2016, we created a multidisciplinary committee, the ERAS Working Group, comprising of participants from neurosurgery, anesthesiology, in-patient and operative nursing, as well as nutrition services departments. All patients were given a complete explanation of the study protocol, drug, and numerical visual analogue scale (VAS) scores during the preoperational evaluation.

After obtaining written and informed consent, 109 adult patients (American Society of anesthesiologists [ASA] physical status I or II, aged 17-75 years) undergoing infratentorial craniotomy between Oct 2016 and March 2018 were enrolled for this study. This study adopted a complete randomized grouping design. Before induction of anesthesia, the nurses responsible for patient allocation randomized the patients using a computer-generated random number table (Microsoft Excel 2013). Patients meeting the inclusion criteria were randomized using opaque sequentially numbered sealed envelopes into two groups: the control group and the ERAS group. Patients who got odd numbers were assigned to the control group, while those who got even numbers were assigned to the ERAS group. Exclusion criteria were intracranial trauma, microvacular decompression, pathology requiring emergency surgery, preoperative disturbance of consciousness, and presence of a confounding condition (e.g., pregnancy) or disease that could potentially impact postoperative recovery (e.g., paralysis, spinal deformity, autoimmune diseases, myocardial infarction, severe infection, liver and renal malfunction, or severe psychological or mental illness).

ERAS protocol and conventional care

This prospective study was conducted to compare postoperative nausea, vomiting, anxiety and sleep quality in patients undergoing infratentorial craniotomy following the implementation of either an ERAS protocol or conventional care. The routine perioperative care of the control group was decided by individual surgeons and anesthetists, based on routine postoperative protocols (Supplementary file). The perioperative care of the ERAS group was managed according to the novel neurosurgical ERAS protocol described in this study. This ERAS protocol was developed based on an extensive review of the recent 
literature investigating evidenced-based perioperative care interventions and successful ERAS programs used in other operations[10]. Our novel ERAS protocol provided a standardized pathway that guided the perioperative management of patients undergoing craniotomy at Tangdu Hospital.

Briefly, the ERAS protocol consists of three main sections: Preoperatively, patients were evaluated and intervened based on the ERAS program that included preoperative counseling, preoperative functional status evaluation, preoperative smoking and alcohol abstinence (at least 2 weeks prior to surgery), mental state assessment, evaluation and prophylactic anti-thrombotic therapy, PONV risk score assessment, preoperative intestinal intervention (Glycerine Enema induction was given for patients with long history of constipation or $\geq 2$ days without defecation.), nutritional assessment (NRS2002, nutritional status assessment, and PG-SGA were applied for nutritional assessment), and preoperative oral carbohydrate loading. During surgery, guidelines for the conduct of surgical and anesthetic management were implemented, including micro-invasive surgery for craniotomy, scalp incision anesthesia, non-opioid analgesia, absorbable skin suture, hypothermia avoidance, and fluid balance. Postoperatively, the pathway provided specific instructions for pain and PONV control, ambulation, urinary drainage, deep vein thrombosis (DVT) prophylaxis, oral intake, etc.

PONV management is a vital component of our ERAS protocol. Preoperative PONV Simple Risk Assessment Scale was used to anticipate the relative risks of each individual. In the ERAS group, the high-risk patients (score $\geq 3$ ) were administered tropisetron (a $5-\mathrm{HT}_{3}$ antagonist, $2 \mathrm{mg}$ ) shortly before extubation for prophylaxis of postoperative vomiting. The patients in the control group did not receive prophylactic tropisetron. Postoperatively, the nausea VAS was used as one of the indications for PONV prevention and treatment in the ERAS group. After the patient was fully conscious, had stable vital signs, was extubated and breathing spontaneously, and could carry out basic commands, all ERAS patients were postoperatively monitored for PONV for $72 \mathrm{~h}$. For patients who had moderate and severe nausea (VAS $\geq 5$ ), tropisetron (2-5 mg, daily) was the first drug of choice. If tropisetron was ineffective, the combination of tropisetron (2-5 mg) and dexamethasone (5 mg), or the combination of tropisetron (2-5 $\mathrm{mg}$ ) and droperidol (0.625-1.25 mg) was given until the VAS was $\leq 5$. In the control group, antiemetics were administered only for patients with vomiting.

In addition, perioperative nutrition management was one of our foremost concerns. In the ERAS group, oral carbohydrate loading (i.e., maltodextrin fructose solution, $400 \mathrm{ml}$ ) was applied 2 hours prior to the surgery. Postoperatively, oral water intake was permitted $4 \mathrm{~h}$ after surgery, while a polymeric nutritional supplement drink was given $8 \mathrm{~h}$ after surgery as tolerated by the patient in the ERAS group. In the control group, patients were allowed to take flow food on the postoperative day (POD) 1-2, semi-liquid food on POD 3-4, and solid food on POD 4-5. Thus, patients in the ERAS group had a shorter duration to first water intake (median 4 hours in ERAS group vs. 10 hours in control group), and first oral solid food intake (median 24 hours vs. 96 hours). Optimization of pain management was also a key element of our ERAS protocol. Local anesthetic administration with ropivacaine $(0.2 \%)$ prior to scalp incision was applied as per our ERAS protocol. Scalp incision closure was achieved by absorbable intradermal running suturing in all ERAS patients. Postoperative morphine and equivalent opioids were not routinely prescribed in our 
patients of both group given their limited efficacy and wide range of side effects, unless a patient's pain VAS score was greater than 7. Instead, nonopioid analgesia strategies such as the administration of intravenous acetaminophen or nonsteroidal antiinflammatory drugs (NSAIDs) were applied according to the intensity of the patient's postoperative VAS score. Postoperatively, we applied same analgesia strategies to both groups patients. Early mobilization and shortened urinary catheterization were encouraged in our ERAS protocol. For deep vein thrombosis (DVT) evaluation and prophylaxis, patients in both groups were encouraged to perform frequent active and passive movement of their lower limbs. Mechanical DVT prophylaxis, which includes compressive stockings and intermittent pneumatic compression pump treatment, was uniformly performed for both groups after surgery. Additionally, many other ERAS elements are not specifically mentioned here. Supplementary file lists all the ERAS elements in detail.

\section{Outcome measurements}

This study was an observer-blinded RCT. Only those who collected and assessed outcomes were blinded. Various parameters were perioperatively assessed by blinded nurses. The same surgical and anesthesia teams performed all procedures. Anesthesia and monitoring were standardized for all patients. At our center, intravenous-inhalation combined anesthesia was adopted for craniotomy patients according to the anesthetist's preference. Atropine and dexamethasone were preoperatively administrated to reduce gland secretion and minimize stress response. Propofol, sufentanil and rocuronium were used for anesthesia induction, while propofol, fentanyl and sevoflurane were used for anesthesia maintenance.

After the operation, all patients were postoperatively observed for $72 \mathrm{~h}$. Vomiting was recorded as either present or absent by direct observation. The time and number of vomiting episodes were recorded. Both vomiting and retching were considered as emetic events. Nausea was scored using a 10-point VAS, with 0 indicating no nausea and 10 indicating the most severe nausea. Upon arrival in the intensive care unit, the patients were asked by the nurse to rate their nausea experience on the VAS device. This process was repeated every $8 \mathrm{~h}$ within $72 \mathrm{~h}$ in the surgical ward. While the patient was asleep, nausea was not assessed. Nausea was defined as a subjectively unpleasant sensation associated with the awareness of the urge to vomit; vomiting was defined as labored, spasmodic, rhythmic contraction of the respiratory muscles with or without expulsion of gastric contents from the mouth [11, 12]. Complete response was regarded as no vomiting or very mild nausea (VAS 0-2). All drugs given for PONV relief were documented.

Subjectively rated anxiety was assessed on a 0 to 10 VAS ( $0=$ very calm; $10=$ very anxious). This parameter was postoperatively evaluated every morning. Subjectively rated quality of sleep, on a 0 to 10 VAS ( 0 = unable to sleep because of anxiety; $10=$ deep and satisfactory sleep), was also postoperatively assessed immediately every morning. All postoperative surgical complications were judged and recorded by the investigator.

Statistical analysis 
The analyses were performed using SPSS (Ver. 19, IBM Corp., Armonk, NY). In neurosurgical patients, a $40 \%$ incidence of PONV after craniotomy was reported[13]. Based on the hypothesis that our ERAS protocol was expected to reduce the postoperative cumulative incidence of vomiting by at least $20 \%$, a sample size of at least 41 patients per arm was calculated to have a power of $80 \%$ and a significance of $5 \%$. To compensate for potential dropouts, 132 patients were enrolled. Interim analysis was planned when the minimal number of the predefined sample size was met.

The cumulative incidence of vomiting for $72 \mathrm{~h}$ after surgery was compared between the two groups using the Cox proportional hazards model. Other categorical variables were analyzed using the Fisher's exact test and Chi-squared test, while continuous variables were analyzed using the t-test. Analysis of the parameters whose values were in large ranges was verified using the Mann-Whitney U-test. Continuous variables were expressed as the mean \pm standard deviation; categorical variables were summarized as counts and percentage. A $p$ value $<0.05$ was deemed to indicate statistical significance.

\section{Results}

\section{Demographics}

A total of 132 patients from our hospital were enrolled in the present study. After exclusion, a total of 105 patients (55 in the control group and 50 in the ERAS group) were included in the analysis (Figure 1). There were no statistically significant differences in age, gender distribution, ASA class, and concomitant diseases between the two groups (Table 1). The relevant details of surgery and tumor pathology are summarized in Table 2. There were no significant differences in primary variables for surgery between the two groups. All patients woke up immediately after the interruption of anesthetics, and were fully conscious.

PONV and the use of antiemetics

The two primary goals of this study were to assess the PONV and antiemetics consumption (Table 3). The preoperative PONV risk assessment was performed on all patients of the two groups. Twenty (40\%) patients in the ERAS group were high-risk individuals of PONV versus 18 (33\%) patients in the control group $(p=0.54)$. Based on the preoperative PONV risk score, the high-risk patients in the ERAS group received PONV prophylaxis (40\%, RR: $74.61,95 \% \mathrm{Cl}: 4.35-1278, \mathrm{p}<0.0001)$. The cumulative incidence of vomiting over the $72 \mathrm{~h}$ post-craniotomy observation period was significantly lower in the ERAS group patients than in the control group patients (Cox proportional test; HR: $0.47,95 \% \mathrm{Cl}: 0.23-0.96,{ }^{*} p=0.03$ ) (Figure 2).

There was no difference in the rate of complete response between the two groups on POD $1(p=0.56)$. However, the rates of complete response were significantly higher in the ERAS group on PODs 2 and 3 (POD 2: 31/50, 62\% in ERAS vs. 22/55, 40\% in control; RR: $2.44,95 \% \mathrm{Cl}: 1.11-5.37, p=0.03$. POD 3: 37/50, $74 \%$ in ERAS vs. $29 / 55,53 \%$ in control; RR: $2.55,95 \% \mathrm{Cl}: 1.11-5.82, \mathrm{p}=0.02)$. Meanwhile, the incidence of vomiting was significantly lower in the ERAS group on PODs 2 and 3 (POD 2: 3/50, 6\% in ERAS vs. 12/55, 
$22 \%$ in control; RR: $0.22,95 \% \mathrm{Cl}: 0.06-0.86, p=0.02$. POD 3: $1 / 50,2 \%$ in ERAS vs. $8 / 55,15 \%$ in control; RR: $0.11,95 \% \mathrm{Cl}: 0.01-0.99, \mathrm{p}=0.03)$.

Additionally, more patients in the ERAS group scored their nausea as mild level on POD 1 as compared to the control group, but the difference was not significant ( $p=0.33$ ). Notably, on PODs 2 or 3 , the nausea score revealed a significantly higher proportion of mild nausea in the ERAS group as compared to the control group (POD 2: 35/50, 70\% in ERAS vs. $25 / 55,45 \%$ in control; RR: $2.80,95 \%$ Cl: $1.25-6.26, p=0.01$. POD 3: $40 / 50,80 \%$ in ERAS vs. $33 / 55,60 \%$ in control; RR: $2.66,95 \% \mathrm{Cl}: 1.10-6.41, p=0.03)$.

Lastly, antiemetics were not more frequently used in the ERAS group than in the control group on the POD $1(p=0.14)$. In addition, on PODs 2 and 3 , there were no significant differences between the two groups, although the nausea score and the vomiting incidence were lower in the ERAS group. Postoperatively, tropisetron, which was regarded as the first-line antiemetic, was given when a patient complained of moderate or serious nausea or if vomiting occurred. The total amount of tropisetron doses per user in each group were almost identical on PODs 1, 2 and 3 (POD 1: $p=0.61$; POD 2: $p=0.63$; POD 3: $p=0.60$ ).

Postoperative anxiety and sleep quality

The two secondary goals of this study were to assess the postoperative anxiety level and the associated sleep disturbances (Table 4). During PODs 0 to 3, the ERAS group patients had a self-rated anxiety level that was significantly lower than that of the control group patients (POD 1: $p=0.01 ; P O D 2: p<0.01 ; P O D$ $3: p=0.01)$. The quality of sleep on PODs 1,2 and 3 was rated significantly better by the ERAS group patients (i.e., a higher VAS score) than the controls (POD 1: $p=0.02 ;$ POD 2: $p=0.03 ;$ POD 3: $p=0.03$ ).

Postoperative complications

The characteristics of the surgical complications were similar in the two groups (Table 5). In the present study, there were no serious postoperative complications, such as related death or disturbance of consciousness. One ERAS group patient and two control group patients suffered intracranial hemorrhage, but none of them required reoperation. Neurological deficits occurred in 7 patients from the ERAS group and 11 patients from the control group. Of these patients, most of them were diagnosed to be facial paralysis secondary to acoustic neuroma surgery. All patients were conservatively treated with medication for their complications. Three patients in the ERAS group and two patients in the control group had surgical site infections, and recovered after sterile dressing change and antibiotic treatment. One patient in the control group had an intracranial infection, and recovered after antibiotic treatment and lumbar drainage.

\section{Multivariable associations}

Univariate analysis showed significant association between a lower incidence of vomiting on POD 2 and the following parameters in all patients of the two groups: male, low-risk individuals of PONV, preoperative mouth-rinsing, duration of surgery $<315$ minutes, did not use colloid, and PONV prophylaxis. Multivariate logistic regression model, including variables with $p<0.20$ in the univariate analysis, was 
used to evaluate independent predictors of vomiting on POD 2. The multivariable model is shown in Table 6. High-risk individuals of PONV (RR: $170.609,95 \% \mathrm{Cl}$ : 5.773-5042.077, $\mathrm{p}=0.003$ ), duration of surgery > 315 minutes (RR: 22.611, 95\% Cl: 1.307-391.205, $p=0.032$ ), and use of colloid (RR: $44.161,95 \%$ $\mathrm{Cl}$ : 2.754-708.126, $\mathrm{p}=0.007)$ were significant risk factors for vomiting on POD 2. Preoperative mouthrinsing (RR: $0.007,95 \% \mathrm{Cl}: 0.000-0.331, \mathrm{p}=0.012$ ), and PONV prophylaxis (RR: $0.012,95 \% \mathrm{Cl}: 0.000-$ $0.479, p=0.019$ ) were independent predictors for a lower incidence of vomiting on POD 2 in the multivariate analysis.

\section{Discussion}

The development of a novel, multidisciplinary, evidence-based, neurosurgical ERAS protocol for elective craniotomy was associated with shortened postoperative hospital length of stay and rapid recovery after surgery[14]. We also investigated whether the ERAS protocol was superior to conventional perioperative management in infratentorial craniotomy patients. This was the first randomized, controlled trial to demonstrate that the ERAS protocol for elective infratentorial craniotomy was associated with a significantly lower cumulative incidence of postoperative vomiting, nausea and anxiety, and better postoperative quality of sleep, which led to an accelerated functional recovery. Additionally, the surgical complications were not significantly different between the ERAS and control groups.

PONV is usually defined as any nausea or vomiting occurring within the first 24-72 hours after surgery. Protracted PONV can be extremely distressing to patients and is one of the most common causes of dehydration, acid-base disturbances, and electrolyte imbalance after surgery[15]. The physical act of vomiting may raise intracranial or cerebral intravascular pressure, jeopardizing cerebral perfusion and hemostasis. However, there is a lack of uniform standards in the definitions of "nausea" and "vomiting". In some studies, analysis of PONV is restricted to vomiting, whereas in others, nausea and vomiting are recorded together. Nausea is a subjective sensation requiring activation of neural pathways, which eventually project to areas of the cerebral hemispheres dealing with conscious sensations[16]. Nausea is not always followed by vomiting. In some studies, nausea and vomiting were considered as two different biological phenomena[17], and were separately analyzed. In neurosurgical patients, a $40-80 \%$ incidence of PONV within $24 \mathrm{~h}$ after craniotomy was reported[18, 19]. Moreover, several observational studies demonstrated that infratentorial surgeries were associated with a higher incidence of PONV[2, 20, 21]. For instance, Meng concluded that PONV frequently occurs in adults recovering from retromastoid craniectomy with microvascular decompression of cranial nerves[22]. A possible biological reason for this may be because the surgical area is close to the area postrema (vomiting center)[23]. It receives input from the chemoreceptor trigger zone, vestibular apparatus, cerebellum, and solitary tract nucleus[24]. Inadvertent or unavoidable interference with these areas may cause release of humoral factors that may be stimuli for PONV[2]. Conceivably, infratentorial craniotomy was an especially high-risk factor for PONV in patients undergoing craniotomy.

Our ERAS protocol incorporated recent evidences and expert opinions for effective perioperative PONV management. In our ERAS protocol, preoperative PONV Simple Risk Assessment Scale was applied to 
anticipate the relative risk of each patient, and associated prophylaxis was given based on the result[25]. In a systematic review, the factors considered to have a potential effect on the risk of experiencing PONV included female gender, a history of motion sickness or PONV, nonsmoking status, and use of postoperative opioids[25]. The incidence of PONV with the presence of $0,1,2,3$, or all 4 of these risk factors were $10 \%, 20 \%, 40 \%, 60 \%$, and $80 \%$, respectively[26]. All patients who had the PONV risk $>40 \%$ according to the simplified risk score were evaluated as high-risk populations. In the ERAS group, these high-risk patients were administered tropisetron shortly before extubation for prophylaxis of postoperative vomiting. At present, there is no evidence of any difference in efficacy and harm of different $5-\mathrm{HT}_{3}$ receptor antagonists (tropisetron, ondansetron, granisetron, and dolasetron). Therefore, for the treatment of PONV symptoms, we use the cheapest $5-\mathrm{HT}_{3}$ receptor antagonist (tropisetron) in our hospital pharmacy. $5-\mathrm{HT}_{3}$ receptor antagonists were proven to be effective for the prevention of postoperative emesis after craniotomy, with minimal adverse effects[27-29]. They do not produce sedation, extrapyramidal reactions or drug interactions with other anesthetic drugs. However, when antinausea and anti-vomiting efficacies were separately analyzed, $5-\mathrm{HT}_{3}$ receptor antagonist was found to have a greater anti-vomiting effect than an anti-nausea effect[30]. In contrast, there is more anti-nausea and less anti-vomiting efficacy with the dopamine receptor antagonist (droperidol)[31]. Therefore, there is evidence of an increased antiemetic efficacy with a combination of droperidol and $5-\mathrm{HT}_{3}$ receptor antagonist in the surgical setting[32]. However, in a recent warning by the Food and Drug Administration (FDA), droperidol, when used in antiemetic doses, was associated with prolongation of QTc interval and fatal arrhythmias[23]. The FDA recommended that droperidol should not be used as a first-line therapy. Besides, similar effectiveness was observed when a $5-\mathrm{HT}_{3}$ receptor antagonist was combined with droperidol or dexamethasone[33].

Based on the above, in our ERAS protocol, the intervention measures (nausea score, VAS $\geq 5$ ) were applied for attenuating symptoms according to nausea score after surgery. We tracked both antiemetics use and patients' reporting of nausea. The results showed that more patients in the ERAS group reported mild nausea and complete response (VAS 0-4) on PODs 2 and 3 than in control group (Table 3). The results of this study also showed that our PONV management in the ERAS protocol was associated with a lower cumulative incidence of vomiting after surgery as compared to the conventional care. Although more ERAS patients requested antiemetics in comparison with the control patients on POD 1, there was no statistically significant difference in postoperative tropisetron consumption per user between the two groups. These phenomena could be explained by the efficacy of our PONV management strategy. Based on the use of nausea VAS, more postoperative patients in the ERAS group who needed antiemetics treatment were selected. Our PONV management strategy in the ERAS protocol was effective for preventing PONV on PODs 2 and 3, without any increase in tropisetron dosage per user.

Our ERAS protocol for elective craniotomy focussed on enhanced recovery and shorter hospital stay after surgery[14]. It is well known that craniotomy can substantially disturb the homeostasis of the body and cause extensive surgical stress[34]. Hence, an adequate stress response is advantageous. However, excessive stress can lead to a pathological state. An excessive stress response may predispose the 
patient to an increased risk of cardiovascular and cerebrovascular complications, nutrient malabsorption, and delayed convalescence. Our ERAS strategies, including prophylaxis and management of PONV, DVT, preoperative fasting, incisional local anesthesia, wound closure (Dural, subcutaneous tissue and skin are sutured by absorbable suture), urinary catheter duration, and surgical site pain, are aimed to reduce the stress-related responses in patients after surgery. Less stress response may lead to less inflammation and pain, with subsequently less postoperative vomiting[35, 36]. Scalp nerve block have been showed to reduce VAS pain scores and analgesics requirements after surgery. It is possible that the lower incidence of PONV in the ERAS group was related to less intraoperative remifentanil consumption and postoperative analgesics use. This may be another reason for improving PONV[37]. In addition, multivariate analysis revealed that preoperative mouth-rinsing (Compound Chlorhexidine Gargle) and PONV prophylaxis were independent predictors for a lower incidence of vomiting on POD 2 in all patients

(Table 6). These predictors may be interpreted as the determinants for reducing vomiting on POD 2 in the ERAS group.

There is mounting evidence to suggest that pain and anxiety may also increase the incidence of PONV after surgery $[38,39]$. Our ERAS strategies incorporated recent evidence and expert opinions in designing the analgesic protocols[40,41]. Our previous study showed a significant improvement in pain control after surgery with ERAS implementation[14]. In addition, patients in the ERAS group were able to achieve earlier oral water/food intake, ambulation, and removal of the urinary catheter after surgery. These ERAS strategies could reduce postoperative discomfort and anxiety, and improve sleep quality in patients with infratentorial craniotomy (Table 4). The ERAS patients showed improved mental state. Therefore, it is not surprising that the ERAS patients in this study had a lower incidence of PONV when compared to the control group.

There were no differences in the incidence of postoperative complications between the ERAS and control groups in this study (Table 5). Limited by the case number, these results might not reflect the positive influences of the ERAS protocol in this aspect. However, this study demonstrated the efficacy of the ERAS protocol, without an increased complication risk.

This study had some limitations. First, due to the need for active participation of the patients, this trial could not be blinded in the clinical setting. Blinding of the patients' study arm was employed in this study for those who collected data and assessed outcomes. Second, this study reported data concerning a relatively small number of patients who underwent infratentorial craniotomy. Therefore, further evaluation of the protocol in larger population is warranted in the future.

\section{Conclusion}

Implementation of our ERAS pathway for infratentorial craniotomy was associated with a reduction in postoperative nausea and vomiting, without any increase in the rate of postoperative complications. Future studies are needed to identify the individual interventions that contribute the most to quality of 
recovery in the acute postoperative period, as well as the long-term effects of ERAS implementation on chronic postoperative PONV and antiemetic use.

\section{Abbreviations}

PONV: postoperative nausea and vomiting; ERAS: Enhanced Recovery After Surgery; RCT: randomized control trial; VAS: visual analogue scale; ASA: american society of anesthesiologists; DVT: deep vein thrombosis; PODs: postoperative days; CPA: cerebellopontine angle.

\section{Declarations}

Ethics approval and consent to participate

The experimental protocol was established, according to the ethical guidelines of the Helsinki Declaration and was approved by the institutional human research and ethics committee of Tangdu Hospital. Written informed consent was obtained from all study participants.

Consent for publication

Not applicable.

Availability of data and materials

All data generated or analysed during this study are included in this published article.

Competing interests

The authors declare that they have no known competing financial interests or personal relationships that could have appeared to influence the work reported in this paper.

\section{Funding}

This work was supported by China Natural Science Foundation (81572470 and 81601100 ). The funding body had and will have no involvement in the study design (apart from responding to peer reviewer comments), data collection, analysis or interpretation.

Authors' contributions

Study design: SMH, BC. Data collections: BLL, LY, LFZ, XJ, BFZ, MJL, LM, ZML, JTN, WHL, YFZ, TZ, YFX, LC, LC, XDS, GDG. Data analysis: DL, YW, TZZ, BLL. Writing: DL. All authors critically reviewed the manuscript and agreed on this final version to be submitted to the journal.

Acknowledgments 
Special thanks to Dr. Jinge Li, Mr. Kai Yao for their contributions in the management and coordination of the study. Dr. Xingye Zhang, Dr. Jungong Jing, Dr. Yong Liu have make great effort to the medical care service in this study. We appreciated Ms. Lihui Yang, Ms. Fan Yao, Ms. Ye Gao and Ms. Jing Zhang for their great contributions in nursing service.

\section{References}

[1] Myklejord DJ, Yao L, Liang H, Glurich I. Consensus guideline adoption for managing postoperative nausea and vomiting. WMJ 2012;111:207-213; quiz 214.

[2] Tan C, Ries CR, Mayson K, Gharapetian A, Griesdale DE. Indication for surgery and the risk of postoperative nausea and vomiting after craniotomy: a case-control study. J Neurosurg Anesthesiol 2012;24:325-330.

[3] Gustafsson UO, Scott MJ, Hubner M, et al. Guidelines for Perioperative Care in Elective Colorectal Surgery: Enhanced Recovery After Surgery (ERAS $\left.{ }^{\circledR}\right)$ Society Recommendations: 2018. World J Surg 2019;43:659-695.

[4] Thorell A, MacCormick AD, Awad S, et al. Guidelines for Perioperative Care in Bariatric Surgery: Enhanced Recovery After Surgery (ERAS) Society Recommendations. World J Surg 2016;40:2065-2083.

[5] Kehlet H, Wilmore DW. Multimodal strategies to improve surgical outcome. Am J Surg 2002;183:630-641.

[6] Ljungqvist O, Scott M, Fearon KC. Enhanced Recovery After Surgery: A Review. JAMA Surg 2017;152:292-298.

[7] Cerantola Y, Valerio M, Persson B, et al. Guidelines for perioperative care after radical cystectomy for bladder cancer: Enhanced Recovery After Surgery (ERAS(®)) society recommendations. Clin Nutr 2013;32:879-887.

[8] Jones EL, Wainwright TW, Foster JD, Smith JR, Middleton RG, Francis NK. A systematic review of patient reported outcomes and patient experience in enhanced recovery after orthopaedic surgery. Ann $R$ Coll Surg Eng/2014;96:89-94.

[9] Ota $\mathrm{H}$, Ikenaga $\mathrm{M}$, Hasegawa J, et al. Safety and efficacy of an "enhanced recovery after surgery" protocol for patients undergoing colon cancer surgery: a multi-institutional controlled study. Surg Today 2017;47:668-675.

[10] Hagan KB, Bhavsar S, Raza SM, et al. Enhanced recovery after surgery for oncological craniotomies. J Clin Neurosci 2016;24:10-16.

[11] Dennis K, Zeng L, De Angelis C, et al. A prospective cohort study of patient-reported vomiting, retching, nausea and antiemetic use during neoadjuvant long-course radiation therapy and concurrent 5- 
fluorouracil-based chemotherapy for rectal adenocarcinoma. Clin Trans/ Radiat Oncol 2018;10:42-46.

[12] Babic T, Browning KN. The role of vagal neurocircuits in the regulation of nausea and vomiting. Eur J Pharmacol 2014;722:38-47.

[13] Fabling JM, Gan TJ, El-Moalem HE, Warner DS, Borel CO. A randomized, double-blind comparison of ondansetron versus placebo for prevention of nausea and vomiting after infratentorial craniotomy. $J$ Neurosurg Anesthesiol 2002;14:102-107.

[14] Wang Y, Liu B, Zhao T, et al. Safety and efficacy of a novel neurosurgical enhanced recovery after surgery protocol for elective craniotomy: a prospective randomized controlled trial. J Neurosurg 2018:112.

[15] Horn CC, Wallisch WJ, Homanics GE, Williams JP. Pathophysiological and neurochemical mechanisms of postoperative nausea and vomiting. Eur J Pharmacol 2014;722:55-66.

[16] Kenward H, Pelligand L, Savary-Bataille K, Elliott J. Nausea: current knowledge of mechanisms, measurement and clinical impact. Vet $J$ 2015;203:36-43.

[17] Napadow V, Sheehan JD, Kim J, et al. The brain circuitry underlying the temporal evolution of nausea in humans. Cereb Cortex 2013;23:806-813.

[18] Jain V, Mitra JK, Rath GP, Prabhakar H, Bithal PK, Dash HH. A randomized, double-blinded comparison of ondansetron, granisetron, and placebo for prevention of postoperative nausea and vomiting after supratentorial craniotomy. J Neurosurg Anesthesiol 2009;21:226-230.

[19] Latz B, Mordhorst C, Kerz T, et al. Postoperative nausea and vomiting in patients after craniotomy: incidence and risk factors. J Neurosurg 2011;114:491-496.

[20] Kurita N, Kawaguchi M, Nakahashi K, et al. [Retrospective analysis of postoperative nausea and vomiting after craniotomy]. Masui 2004;53:150-155.

[21] Stadler M, Bardiau F, Seidel L, Albert A, Boogaerts JG. Difference in risk factors for postoperative nausea and vomiting. Anesthesiology 2003;98:46-52.

[22] Meng L, Quinlan JJ. Assessing risk factors for postoperative nausea and vomiting: a retrospective study in patients undergoing retromastoid craniectomy with microvascular decompression of cranial nerves. J Neurosurg Anesthesiol 2006;18:235-239.

[23] Gan TJ. Postoperative nausea and vomiting--can it be eliminated. JAMA 2002;287:1233-1236.

[24] Elwood C, Devauchelle P, Elliott J, et al. Emesis in dogs: a review. J Small Anim Pract 2010;51:4-22.

[25] Eberhart LH, Morin AM, Kranke P, Missaghi NB, Durieux ME, Himmelseher S. Prevention and control of postoperative nausea and vomiting in post-craniotomy patients. Best Pract Res Clin Anaesthesiol 
2007;21(4):575-93.

[26] Weilbach C, Rahe-meyer N, Raymondos K, Weissig A, Scheinichen D, Piepenbrock S. Postoperative nausea and vomiting (PONV) : usefulness of the Apfel-score for identification of high risk patients for PONV. Acta Anaesthesiol Belg 2006;57:361-363.

[27] Herrstedt J. The latest consensus on antiemetics. Curr Opin Oncol 2018;30:233-239.

[28] Neufeld SM, Newburn-Cook CV. The efficacy of 5-HT3 receptor antagonists for the prevention of postoperative nausea and vomiting after craniotomy: a meta-analysis. J Neurosurg Anesthesiol 2007;19:10-17.

[29] Rojas C, Raje M, Tsukamoto T, Slusher BS. Molecular mechanisms of 5-HT(3) and NK(1) receptor antagonists in prevention of emesis. Eur J Pharmacol 2014;722:26-37.

[30] Andrews PL, Sanger GJ. Nausea and the quest for the perfect anti-emetic. Eur J Pharmacol 2014;722:108-121.

[31] Perkins J, Ho JD, Vilke GM, DeMers G. American Academy of Emergency Medicine Position Statement: Safety of Droperidol Use in the Emergency Department. J Emerg Med 2015;49:91-97.

[32] Matsota P, Angelidi M, Pandazi A, Tzirogiannis KN, Panoutsopoulos Gl, Kostopanagiotou G. Ondansetron-droperidol combination vs. ondansetron or droperidol monotherapy in the prevention of postoperative nausea and vomiting. Arch Med Sci 2015;11:362-370.

[33] Habib AS, El-Moalem HE, Gan TJ. The efficacy of the 5-HT3 receptor antagonists combined with droperidol for PONV prophylaxis is similar to their combination with dexamethasone. A meta-analysis of randomized controlled trials. Can J Anaesth 2004;51:311-319.

[34] Li HT, Zhao ZH, Ding HY, Wang LX, Cao Y. Effect of craniotomy on oxidative stress and its effect on plasma L-carnitine levels. Can J Physiol Pharmacol 2014;92:913-916.

[35] Odom-Forren J, Rayens MK, Gokun Y, et al. The Relationship of Pain and Nausea in Postoperative Patients for 1 Week After Ambulatory Surgery. Clin J Pain 2015;31:845-851.

[36] Ren L, Zhu D, Wei Y, et al. Enhanced Recovery After Surgery (ERAS) program attenuates stress and accelerates recovery in patients after radical resection for colorectal cancer: a prospective randomized controlled trial. World J Surg 2012;36:407-414.

[37] Yang X, Ma J, Li K, Chen L, Dong R, Lu Y, et al. A comparison of effects of scalp nerve block and local anesthetic infiltration on inflammatory response, hemodynamic response, and postoperative pain in patients undergoing craniotomy for cerebral aneurysms: a randomized controlled trial. BMC Anesthesiol 2019;19(1):91. 
[38] de Boer HD, Detriche O, Forget P. Opioid-related side effects: Postoperative ileus, urinary retention, nausea and vomiting, and shivering. A review of the literature. Best Pract Res Clin Anaesthesiol 2017;31:499-504.

[39] Yap KY, Low XH, Chui WK, Chan A, Onco-Informatics Group. Computational prediction of state anxiety in Asian patients with cancer susceptible to chemotherapy-induced nausea and vomiting. $J$ Clin Psychopharmacol 2012;32:207-217.

[40] Dilmen OK, Akcil EF, Tunali Y, et al. Postoperative analgesia for supratentorial craniotomy. Clin Neurol Neurosurg 2016;146:90-95.

[41] Guilfoyle MR, Helmy A, Duane D, Hutchinson PJ. Regional scalp block for postcraniotomy analgesia: a systematic review and meta-analysis. Anesth Analg 2013;116:1093-1102.

\section{Tables}

Table 1. Patient demographics

\begin{tabular}{lccc}
\hline \multicolumn{1}{c}{ Parameter } & $\begin{array}{c}\text { Control group } \\
(\mathbf{n}=\mathbf{5 5})\end{array}$ & $\begin{array}{c}\text { ERAS group } \\
(\mathbf{n}=50)\end{array}$ & P value \\
& $48.7 \pm 12.5$ & $47.0 \pm 13.0$ & 0.51 \\
\hline Mean age, years & $20(36 \%)$ & $21(42 \%)$ & 0.68 \\
Gender & $35(64 \%)$ & $29(58 \%)$ & \\
Male, n(\%) & $23.0 \pm 2.7$ & $23.3 \pm 2.9$ & 0.55 \\
Female, n(\%) & $18(33 \%)$ & $19(38 \%)$ & 0.57 \\
Mean BMI, kg/m2 & $37(67 \%)$ & $31(62 \%)$ & \\
ASA classification & $8(15 \%)$ & $10(20 \%)$ & 0.61 \\
ASA I, n(\%) & $10(18 \%)$ & $12(24 \%)$ & 0.48 \\
ASA II, n(\%) & $1(2 \%)$ & $3(6 \%)$ & 0.35 \\
Concomitant diseases & $3(5 \%)$ & $5(1 \%)$ & 0.47 \\
Cardiac/hypertension, n(\%) & $7(1 \%)$ & 0.45 \\
Smoker, n(\%) & $7(20 \%)$ & $7(14 \%)$ & 0.76 \\
Liver/gall bladder, n(\%) & $7(13 \%)$ & $5(10 \%)$ & 0 \\
Lung, n(\%) & & & \\
Diabetes mellitus, n(\%) & & & \\
Miscellaneous, n(\%) & &
\end{tabular}

Data are expressed as mean \pm SD or number of patients (\%). P value indicates the comparison between control group and ERAS group. ASA, American Society Anesthesiologists. 


\section{Table 2. Surgery characteristics}

\begin{tabular}{lccc}
\hline \multicolumn{1}{c}{ Parameter } & $\begin{array}{c}\text { Control group } \\
(\mathbf{n}=\mathbf{5 5 )}\end{array}$ & $\begin{array}{c}\text { ERAS group } \\
(\mathbf{n}=\mathbf{5 0 )}\end{array}$ & P value \\
\hline Infratentorial approach & $10(18 \%)$ & $9(18 \%)$ & 0.64 \\
CPA meningioma, n(\%) & $10(18 \%)$ & $12(24 \%)$ & \\
Acoustic neuroma, n(\%) & $4(7 \%)$ & $3(6 \%)$ & \\
Trigeminal neuroma, n(\%) & $17(31 \%)$ & $15(30 \%)$ & \\
Glioma, n(\%) & $6(11 \%)$ & $5(10 \%)$ & \\
Hemangioblastoma, n(\%) & $8(15 \%)$ & $6(12 \%)$ & \\
CPA cholesteatoma, n(\%) & $3.1 \pm 1.0$ & $2.9 \pm 1.0$ & 0.30 \\
Tumor size, cm & $15(27 \%)$ & $10.2 \pm(20 \%)$ & 0.52 \\
Mean duration of surgery, minutes & $322.9 \pm 125.5$ & 0.49 \\
Blood loss > 300ml, n(\%) & $7(13 \%)$ & $5(10 \%)$ & 0.76 \\
Blood transfusion, n(\%) & $20(36 \%)$ & $19(38 \%)$ & 1.00 \\
Use of colloid, n(\%) & & & \\
\hline
\end{tabular}

Data are expressed as mean \pm SD or number of patients (\%). P value indicates the comparison between control group and ERAS group. CPA, cerebellopontine angle. 
Table 3. Postoperative nausea and vomiting outcomes

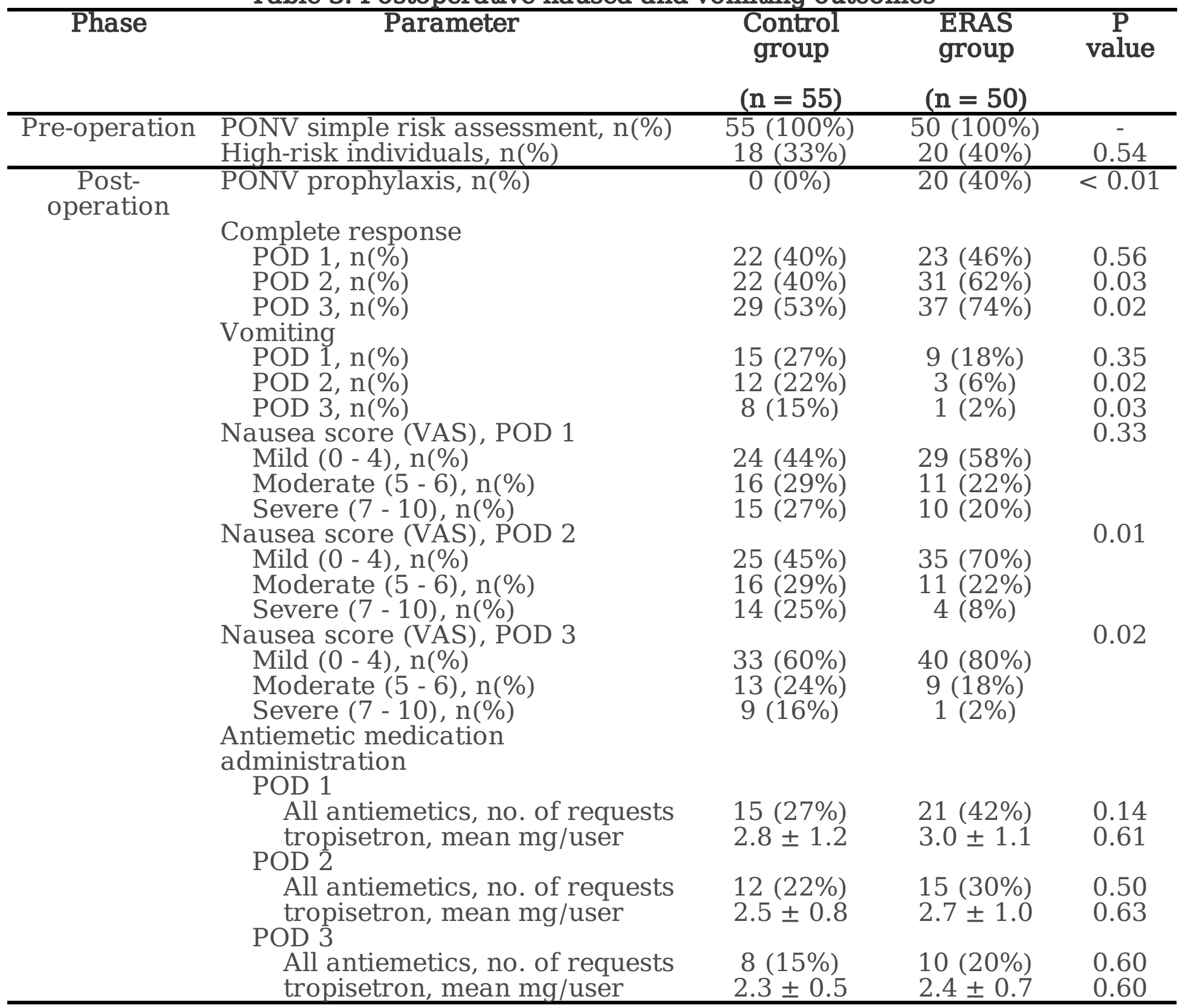

Data are expressed as number of patients (\%). P value indicates the comparison between control group and ERAS group. POD, Postoperative day; PONV, postoperative nausea and vomiting; VAS, Visual Analogue Scale. 
Table 4. Immediate postoperative anxiety level and sleep quality Parameter

Control group ERAS group P value

\begin{tabular}{cccc} 
& $(\mathbf{n}=\mathbf{5 5})$ & $(\mathbf{n}=\mathbf{5 0 )}$ & \\
\hline Anxiety level, mean VAS score & & & \\
POD 1 & $6.5 \pm 1.9$ & $5.6 \pm 1.7$ & 0.01 \\
POD 2 & $5.7 \pm 2.0$ & $4.8 \pm 1.7$ & $<0.01$ \\
POD 3 & $3.9 \pm 1.7$ & $3.1 \pm 1.4$ & 0.01 \\
Sleep quality, mean VAS score & & & \\
POD 1 & $6.5 \pm 1.3$ & $7.2 \pm 1.5$ & 0.02 \\
POD 2 & $5.4 \pm 1.2$ & $5.9 \pm 1.3$ & 0.03 \\
POD 3 & $4.0 \pm 1.3$ & $4.5 \pm 1.3$ & 0.03 \\
\hline
\end{tabular}

Data are expressed as mean \pm SD. P value indicates the comparison between control group and ERAS group. POD, Postoperative day; VAS, Visual Analogue Scale.

Table 5. Postoperative complications

\begin{tabular}{|c|c|c|c|}
\hline Parameter & $\begin{array}{l}\text { Control } \\
\text { group } \\
(\mathrm{n}=55)\end{array}$ & $\begin{array}{l}\text { ERAS } \\
\text { group } \\
(n=50)\end{array}$ & P value \\
\hline Mortality, n(\%) & $0(0 \%)$ & $0(0 \%)$ & - \\
\hline Disturbance of consciousness, n(\%) & $0(0 \%)$ & $0(0 \%)$ & \\
\hline Intracranial infection, $\mathrm{n}(\%)$ & $1(2 \%)$ & $0(0 \%)$ & 1.00 \\
\hline Hemorrhage*, $n(\%)$ & $2(4 \%)$ & $1(2 \%)$ & 1.00 \\
\hline Cerebral infarction, n(\%) & $0(0 \%)$ & $0(0 \%)$ & \\
\hline Neuro deficits**, $n(\%)$ & $11(20 \%)$ & $7(14 \%)$ & 0.44 \\
\hline Aspiration, n(\%) & $0(0 \%)$ & $0(0 \%)$ & \\
\hline $\begin{array}{l}\text { Surgical site infection/ subcutaneous effusion, } \\
\mathrm{n}(\%)\end{array}$ & $2(4 \%)$ & $3(6 \%)$ & 0.66 \\
\hline
\end{tabular}

Data are expressed as number of patients (\%). P value indicates the comparison between control group and ERAS group. * Hemorrhage refers to small amount of epidural hematoma or surgical area hemorrhage, not including intracranial hemorrhage which needs re-operation. ** Neuro deficits refers to cranial-nerve palsies after operation, including facial paralysis, ophthalmoplegia, trigeminal nerve injury, etc.

Table 6. Multivariate logistic regression for predictors of postoperative vomiting on POD 2 in all patients

\begin{tabular}{lll}
\hline Variable & \multicolumn{1}{c}{ RR (95\% CI) } & P value \\
\hline Gender & $8.930(0.957-83.347)$ & 0.055 \\
High-risk individuals of PONV & $170.609(5.773-5042.077)$ & 0.003 \\
Preoperative mouth-rinsing & $0.007(0.000-0.331)$ & 0.012 \\
Duration of surgery $>$ 315 minutes & $22.611(1.307-391.205)$ & 0.032 \\
Use of colloid & $44.161(2.754-708.126)$ & 0.007 \\
PONV prophylaxis & $0.012(0.000-0.479)$ & 0.019 \\
\hline
\end{tabular}

Abbreviations: 
POD: Postoperative day

\section{Figures}

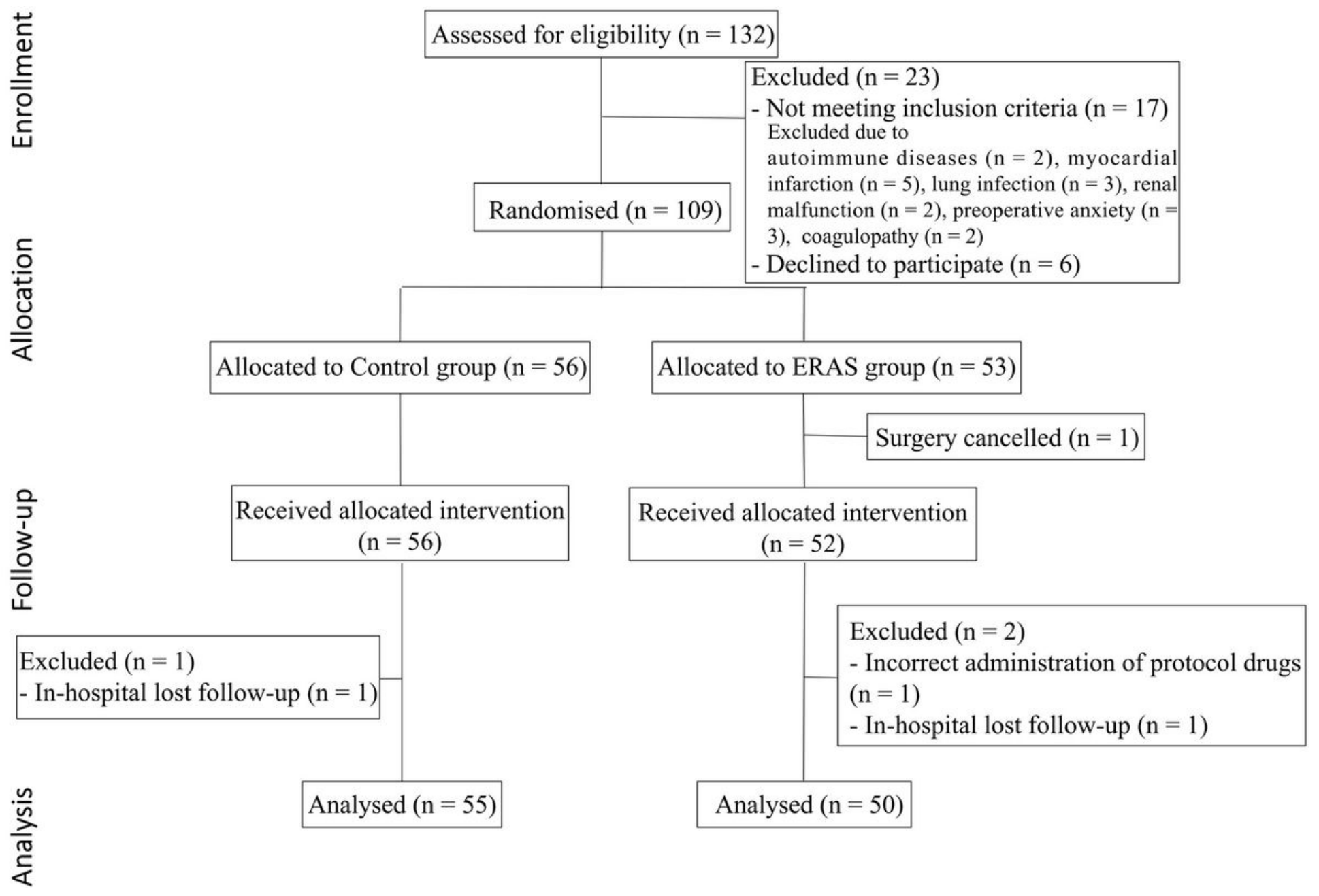

\section{Figure 1}

Flow diagram of CONSORT study design. 


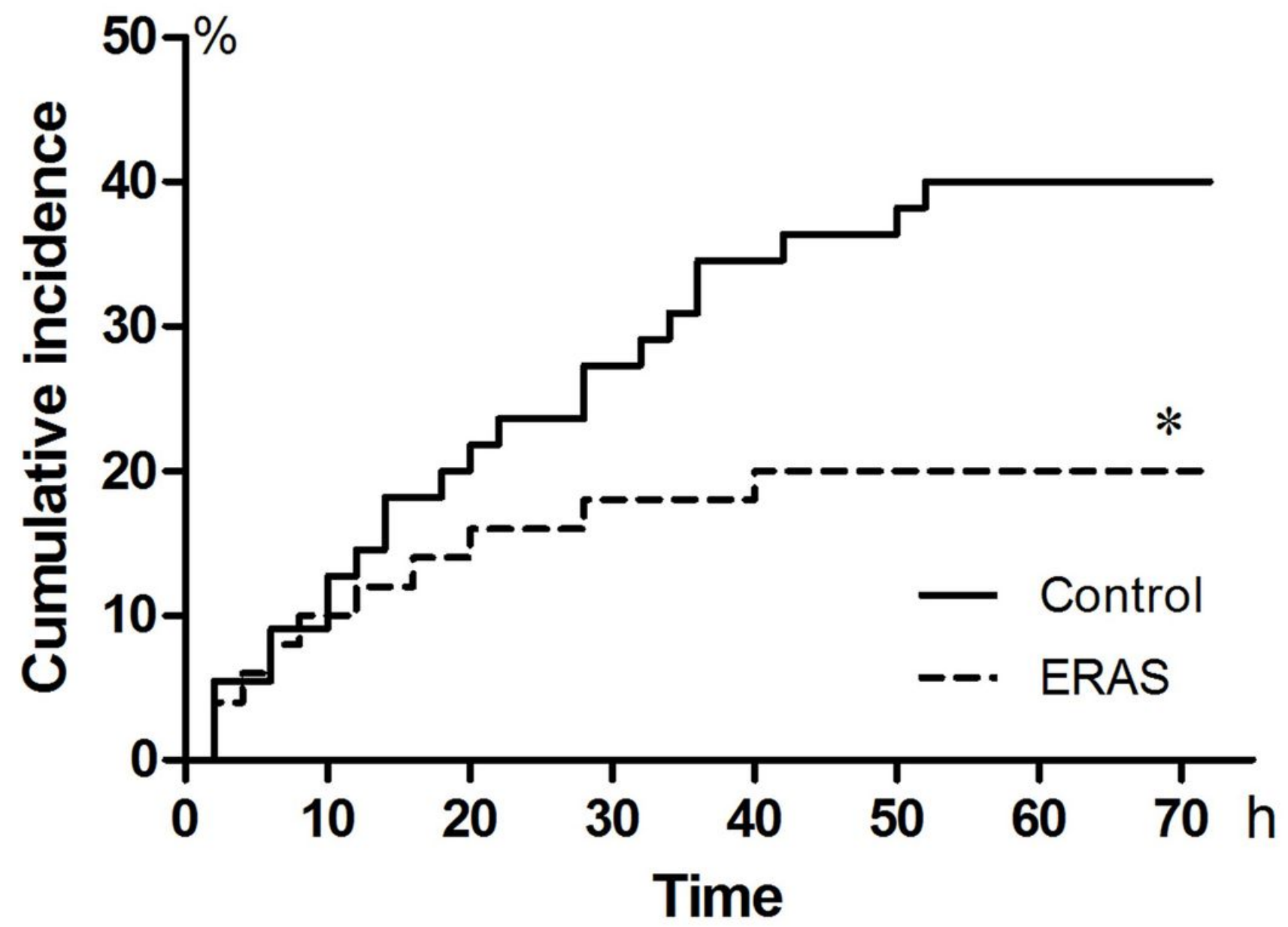

Figure 2

The cumulative incidence of vomiting in patients of the ERAS group and the control group. The cumulative incidence of vomiting over the $72 \mathrm{~h}$ post-craniotomy observation period was significantly lower in the ERAS group patients than in the control group patients.

\section{Supplementary Files}

This is a list of supplementary files associated with this preprint. Click to download.

- Supplementaryfile.doc 